(1)

CrossMark

\title{
Is low level of vitamin D a marker of poor health, or a cause?
}

\author{
Om P. Kurmi (i) \\ Affiliation: Population Health Research Institute, Dept of Medicine, McMaster University, Hamilton, ON, \\ Canada. \\ Correspondence: Om P. Kurmi, Population Health Research Institute, Dept of Medicine, McMaster University, \\ 237 Barton Street East, Hamilton, Ontario L8L 2X2, Canada. E-mail: kurmiodmcmaster.ca
}

@ERSpublications

Vitamin D deficiency is associated with a number of poor health outcomes but the reported findings on incidence of lung cancer and mortality are inconsistent. The current study does not support a causal link between vitamin D and lung cancer. http://ow.ly/NrAd30k94Oq

Cite this article as: Kurmi OP. Is low level of vitamin D a marker of poor health, or a cause? Eur Respir J 2018; 51: 1800931 [https://doi.org/10.1183/13993003.00931-2018].

Vitamin D has long been recognised for its role in maintaining good skeletal health in both adults and children, including the rapid bone mineral accrual in infants, and prevention of rickets in children and osteomalacia in adults $[1,2]$. Studies suggest that vitamin $\mathrm{D}$, an essential hormone ingested from the diet (food and supplements) or synthesised in the skin when exposed to UV-B radiation, regulates calcium, phosphorous and bone metabolism, promotes skeletal muscle strength, inhibits cell proliferation, promotes cell differentiation, reduces inflammation, modulates the renin-angiotensin system, and influences glucose metabolism [3, 4]. Vitamin D obtained from the above sources is the biologically inactive and after undergoing first enzymatic hydroxylation reactions in the liver forms 25-hydroxyvitamin D (25(OH)D), an intermediate product which is stored and circulated. Only a small fraction of $25(\mathrm{OH}) \mathrm{D}$ is then converted to the physiologically active hormone, calcitriol (1,25-dihydroxyvitamin D) following second reaction in the kidney [5,6]. Although people living in low latitudes (e.g. large parts of Asia and Africa) are exposed to abundant sunlight and should therefore, in theory, have low risk of vitamin D deficiency and related morbidities, the evidence, however, suggests the contrary; previous studies have reported high prevalence of vitamin D deficiency in these regions, likely to be contributed by other factors including skin complexion, limited outdoor exposure, vegetarian diet, and poorly implemented (or lack of) vitamin D food fortification programmes [7]. Worldwide it is estimated that up to one billion children and adults are vitamin $\mathrm{D}$ deficient (plasma $25(\mathrm{OH}) \mathrm{D}<20 \mathrm{ng} \cdot \mathrm{mL}^{-1}$, with the preferred range of $25(\mathrm{OH}) \mathrm{D}$ concentration being 40-60 ng. $\mathrm{mL}^{-1}$ ) [4]. Many developed countries, including Canada and USA, have been fortifying milk with Vitamin D to maintain the recommended daily intake level of vitamin D [8]. Even so, there is a growing concern in Europe that the high prevalence of low vitamin D intake is causing vitamin D deficiency [9].

More recently, a large number of epidemiological studies have reported that vitamin D may have extraskeletal effects such that insufficiency in vitamin D may be associated with increased risks of respiratory infections and diseases, cancer, cardiovascular diseases, autoimmune disorders, diabetes, dementia, depression and adverse pregnancy outcomes [10-12]. Understandably these findings have generated considerable interest over the past few decades within the medical community and indeed the public, as an increasing number of people (possibly encouraged by the media or advertisements) purchase over-the-counter supplements without knowing whether they are deficient or not. Vitamin D is believed to have important antiproliferative and prodifferentiation properties [3]. A randomised controlled trial (RCT) 
that used a high dose (1000 IU per day) of vitamin D showed a large beneficial effect (60\% reduction) on incident cancer [13]. However, prevailing guidelines have suggested that the daily dose in elderly individuals should not exceed $3000 \mathrm{IU}$ and serum levels of $25(\mathrm{OH}) \mathrm{D}$ not more than $40-45 \mathrm{ng} \cdot \mathrm{mL}^{-1}(100-$ $112 \mathrm{nmol} \cdot \mathrm{L}^{-1}$ ) [14]. There is also a genuine concern that increasing vitamin $\mathrm{D}$ level by increased exposure to sunlight or UV-B tanning are more likely to increase skin photo-ageing and carcinogenesis. Observational studies have also shown a relationship between sufficient vitamin D status and lower risks of cancer [15-17], including lung cancer [18]. Despite due care taken to improve the design and analysis of observational studies (by controlling for all known potential confounders) reverse causation and unmeasured confounding cannot be completely ruled out, leading to bias and spurious findings [19]. Mendelian randomisation, with the ability to infer causality, has therefore become the method of choice in analysing observational data [19].

In this issue of the European Respiratory Journal, SUN et al. [20] report prospective results regarding vitamin D and lung cancer using a Mendelian randomisation approach. The study followed up 54580 individuals ( $\geqslant 20$ years of age at baseline) from the second survey of Nord-Trondelag Health Study (HUNT2) for a median of 18 years, during which 676 incident lung cancer cases were documented. Approximately $10 \%$ of the participants $(n=5546)$ were randomly selected for serum $25(\mathrm{OH}) \mathrm{D}$ measurement. Three single nucleotide polymorphisms (rs2282679 (GC), rs12785878 (NADSYN1/DHCR7), rs10741657 (CYP2R1)), located in or near the genes for vitamin D synthesis and metabolism and identified from two previous genome-wide association studies [21, 22] were used to create allele scores. In contrast to two recent meta-analyses [23, 24] of non-Mendelian randomisation studies, SuN et al. [20] reported no significant association between the vitamin D increasing alleles of rs2282679, rs12785878, rs10741657 and the overall risk of lung cancer incidence or any of the three histologic types, refuting the causal association suggested previously. In one of the meta-analyses [23] a 28\% reduced risk for overall lung cancer and further reduction in risk for higher $25(\mathrm{OH}) \mathrm{D}$ levels was reported but there was no significant role on survival. On closer scrutiny, only two out of eight studies that were included for calculating the pooled effect size for overall lung cancer had reported significant risk reduction. In the other meta-analysis [17] that included RCTs of vitamin D supplementation (with at least 1 year of follow-up and participants aged $\geqslant 60$ years) there was no evidence to suggest that vitamin $D$ supplementation reduces the incidence of cancer or cancer mortality. It is interesting to note that in the same study population (HUNT) but using a case-cohort analysis approach, lower 25(OH)D levels were associated with lower risk of adenocarcinoma in overweight/obese individuals [25], highlighting the importance of bias due to various confounding. The strength of this paper is the use of a Mendelian randomisation approach but it also has several limitations that have been highlighted by the authors.

There are a number of trials currently being carried out on the effects of vitamin D supplementation, with the majority being conducted in Northern America and Europe, but none in the low income countries, particularly South Asia, where more men and women are reported to be vitamin D deficient [26]. This calls for an investigation that compares the health outcomes among vitamin $\mathrm{D}$ deficient and non-deficient populations in Asia. We also do not have enough data on the combined effect of smoking and other established risk factors, such as exposure to smoke from solid fuel burning, which is a common practice in mainly rural areas where high deficiency in vitamin $\mathrm{D}$ level is reported $[7,26]$. Lung function and vitamin $\mathrm{D}$ levels are lower in smoking populations and it is postulated that low vitamin D level is associated with impaired lung function, but whether there is any joint effect of low vitamin level and smoking on lung function is unclear. A matched case-control study did not find significant association between vitamin D level and decline in lung function [27]. Impaired lung function is a predictor of all-cause and cause-specific mortality, including lung cancer [28]. However, it is uncertain if increasing plasma $25(\mathrm{OH}) \mathrm{D}$ level could improve (or at least slow down the decline of) lung function and chronic obstructive pulmonary disease (COPD) comorbidities. A secondary analysis of the data collected from an RCT suggested that there was no relationship between baseline $25(\mathrm{OH}) \mathrm{D}$ and time to first acute exacerbation of COPD or hospitalisation due to COPD [29], and this has been supported by findings from a recent meta-analysis [30].

The current study was carried out in participants of European ancestry in a high-latitude setting. It would be interesting to see if the results can be replicated in a larger sample and one of different ethnicity, particularly in individuals following different diets and exposed to high levels of indoor and outdoor environmental pollutants. It is also essential to identify a safe limit for intake of vitamin D and show whether a higher dose of vitamin D improves overall health through prospective studies, particularly in vitamin D deficient areas.

Acknowledgements: I am grateful to S. Sadhra (University of Birmingham) and K.B.H. Lam (University of Oxford) for their helpful comments on this editorial.

Conflict of interest: None declared. 


\section{References}

1 Uday S, Högler W. Prevention of rickets and osteomalacia in the UK: political action overdue. Arch Dis Child 2018; in press [https://doi.org/10.1136/archdischild-2018-314826].

2 Gallo S, Comeau K, Vanstone C, et al. Effect of different dosages of oral vitamin D supplementation on vitamin D status in healthy, breastfed infants: a randomized trial. JAMA 2013; 309: 1785-1792.

3 Giovannucci E, Liu Y, Rimm EB, et al. Prospective study of predictors of vitamin D status and cancer incidence and mortality in men. J Natl Canc Inst 2006; 98: 451-459.

$4 \quad$ Holick MF. Vitamin D deficiency. N Engl J Med 2007; 357: 266-281.

5 Ross AC. The 2011 report on dietary reference intakes for calcium and vitamin D. Public Health Nutr 2011; 14 938-949.

6 Milešević J, Samaniego L, Kiely M, et al. Specialized food composition dataset for vitamin D content in foods based on European standards: application to dietary intake assessment. Food Chem 2018; 240: 544-549.

7 Goswami R, Gupta N, Goswami D, et al. Prevalence and significance of low 25-hydroxyvitamin D concentrations in healthy subjects in Delhi. Am J Clin Nutr 2000; 72: 472-475.

8 Holick MF, Shao Q, Liu WW, et al. The vitamin D content of fortified milk and infant formula. $N$ Engl J Med 1992; 326: 1178-1181.

9 Spiro A, Buttriss JL. Vitamin D: an overview of vitamin D status and intake in Europe. Nut Bull 2014; 39: 322-350.

10 Turner AM, McGowan L, Millen A, et al. Circulating DBP level and prognosis in operated lung cancer: an exploration of pathophysiology. Eur Respir J 2013; 41: 410-416.

11 Autier P, Boniol M, Pizot C, et al. Vitamin D status and ill health: a systematic review. Lancet Diabetes Endocrinol 2014; 2: 76-89.

12 Pludowski P, Holick MF, Pilz S, et al. Vitamin D effects on musculoskeletal health, immunity, autoimmunity, cardiovascular disease, cancer, fertility, pregnancy, dementia and mortality-a review of recent evidence. Autoimmun Rev 2013; 12: 976-989.

13 Lappe JM, Travers-Gustafson D, Davies KM, et al. Vitamin D and calcium supplementation reduces cancer risk: results of a randomized trial. Am J Clin Nutr 2007; 85: 1586-1591.

14 Gallagher JC. Vitamin D and falls - the dosage conundrum. Nat Rev Endocrinol 2016; 12: 680-684.

15 Garland CF, Gorham ED, Mohr SB, et al. Vitamin D and prevention of breast cancer: pooled analysis. J Steroid Biochem Mol Biol 2007; 103: 708-711.

16 Gorham ED, Garland CF, Garland FC, et al. Optimal vitamin D status for colorectal cancer prevention: a quantitative meta analysis. Am J Prevent Med 2007; 32: 210-216.

17 Goulão B, Stewart F, Ford JA, et al. Cancer and vitamin D supplementation: a systematic review and meta-analysis. Am J Clin Nutr 2018; 107: 652-663.

18 Wang X, Cui J, Gu J, et al. Plasma 25-hydroxyvitamin D deficiency is associated with the risk of non-small cell lung cancer in a Chinese population. Cancer Biomark 2015; 15: 663-668.

19 Smith GD, Ebrahim S. Mendelian randomization: prospects, potentials, and limitations. Int J Epidemiol 2004; 33: $30-42$.

20 Sun Y-Q, Brumpton BM, Bonilla C, et al. Serum 25-hydroxyvitamin D levels and risk of lung cancer and histologic types: a Mendelian randomisation analysis of the HUNT study. Eur Respir J 2018; 51: 1800329.

21 Ahn J, Yu K, Stolzenberg-Solomon R, et al. Genome-wide association study of circulating vitamin D levels. Hum Mol Genet 2010; 19: 2739-2745.

22 Wang TJ, Zhang F, Richards JB, et al. Common genetic determinants of vitamin D insufficiency: a genome-wide association study. Lancet 2010; 376: 180-188.

23 Liu J, Dong Y, Lu C, et al. Meta-analysis of the correlation between vitamin D and lung cancer risk and outcomes. Oncotarget 2017; 8: 81040-81051.

24 Zhang L, Wang S, Che X, et al. Vitamin D and lung cancer risk: a comprehensive review and meta-analysis. Cell Physiol Biochem 2015; 36: 299-305.

25 Sun YQ, Langhammer A, Wu C, et al. Associations of serum 25-hydroxyvitamin D level with incidence of lung cancer and histologic types in Norwegian adults: a case-cohort analysis of the HUNT study. Eur J Epidemiol 2018; 33: 67-77.

26 Harinarayan CV, Ramalakshmi T, Prasad UV, et al. High prevalence of low dietary calcium, high phytate consumption, and vitamin D deficiency in healthy south Indians. Am J Clin Nutr 2007; 85: 1062-1067.

27 Kunisaki KM, Niewoehner DE, Singh RJ, et al. Vitamin D status and longitudinal lung function decline in the Lung Health Study. Eur Respir J 2011; 37: 238-243.

28 Kurmi OP, Li L, Davis KJ. Excess risk of major vascular diseases associated with airflow obstruction: a 9-year prospective study of 0.5 million Chinese adults. Int J Chron Obstruct Pulmon Dis 2018; 13: 855-865.

29 Kunisaki KM, Niewoehner DE, Connett JE, et al. Vitamin D levels and risk of acute exacerbations of chronic obstructive pulmonary disease: a prospective cohort study. Am J Respir Crit Care Med 2012; 185: 286-290.

30 Zhu M, Wang T, Wang C, et al. The association between vitamin D and COPD risk, severity, and exacerbation: an updated systematic review and meta-analysis. Int J Chron Obstruct Pulmon Dis 2016; 11: 2597-2607. 